\title{
Isolation of Coagulase-Negative Staphylococcus Spp. and Kocuria Varians in Pure Culture from Tissues of Cases of Mortalities in Parrots in Grenada, West Indies
}

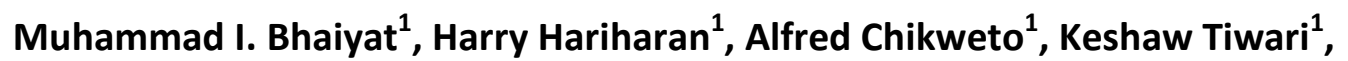 \\ Ravindra N. Sharma ${ }^{1}$ and Yoshiyasu Kobayashi ${ }^{2}$ \\ ${ }^{1}$ Pathobiology Academic Program, School of Veterinary Medicine, St. George's University, Grenada, \\ West Indies \\ ${ }^{2}$ Laboratory of Veterinary Pathology, Division of Pathobiological Science, Department of Basic \\ Veterinary Medicine, Obihiro University of Agriculture and Veterinary Medicine, Hokkaido, Japan
}

Correspondence should be addressed to: Muhammad I Bhaiyat; mibhaiyat@sgu.edu

Received 3 June 2013; Accepted 23 June 2013; Published 18 October 2013

Academic Editor: Walter Tarello

Copyright (C) 2013 Muhammad I. Bhaiyat, Harry Hariharan, Alfred Chikweto, Keshaw Tiwari, Ravindra N. Sharma and Yoshiyasu Kobayashi. Distributed under Creative Commons CC-BY 3.0

\begin{abstract}
Approximately 30 psittacine birds, including African grey parrots and ring-necked parrots died during a period of 1-2 months in 2012 in the parish of St.David, Grenada. At necropsy, the liver showed swelling with red to tan mottling, and there was diffuse pulmonary congestion. Tissues from 8 birds were examined for bacteria by culture. Pure cultures of coagulase-negative staphylococci in moderate to heavy amounts were isolated from almost all tissues, including liver, lung, and spleen. Only 7 isolates could be identified to species level. Liver, lung and intestines of 2 birds were positive for Staphylococcus lentus, and one spleen sample from a single bird was positive for Kocuria varians. Antimicrobials drug susceptibility tests against 10 drugs showed susceptibility to all drugs except for the tetracycline resistance in one isolate. Significance of these findings is discussed in view of the negative results for Pacheco's disease which was originally suspected.
\end{abstract}

Keywords: psittacine birds, coagulase-negative staphylococci, Kocuria varians.

\section{Introduction}

Mortalities in psittacine birds have been reported due to viral diseases such as Pacheco's disease herpesviruses (Bistyak et al., 2007, Barao da Cunha et al., 2007), and bacterial diseases such as chlamydiosis (Raso Tde et al., 2004), and salmonellosis
(Ward et al., 2003). Although uncommon, deaths in these birds may also occur due to Gram-positive bacteria, including Erysipelothrix rhusiopathiae (GalindoCardiel et al., 2012), Lactobacillus jensenii (Foldenauer et al., 2009), and Staphylococcus aureus (Hermans et al., 2000). The role of staphylococcus species

Cite this Article as: Muhammad I. Bhaiyat, Harry Hariharan, Alfred Chikweto, Keshaw Tiwari, Ravindra N. Sharma and Yoshiyasu Kobayashi (2013), "Isolation of Coagulase-Negative Staphylococcus Spp. and Kocuria Varians in Pure Culture from Tissues of Cases of Mortalities in Parrots in Grenada, West Indies," International Journal of Veterinary Medicine: Research \& Reports, Vol. 2013 (2013), Article ID 149634, DOI: $10.5171 / 2013.149634$ 
other than $S$. aureus is less clear. Scanty reports include septicemia in a lovebird, and bacteremia in birds of prey due to coagulase-negative staphylococci (Bounous et al., 1989, Tarello, 2001). We report here isolation of staphylococci and a related bacterium Kocuria varians in large numbers in pure culture from tissues of several psittacine birds.

\section{Materials and Methods}

In early 2012, about 30 psittacine birds, including African grey parrots and ring necked parrots died in the parish of St. David, Grenada during a 1-2 month period. These were from a flock of approximately 200 birds housed in cages. The deaths were sporadic, but in the last week of February 2012, five birds were found dead at one time. Eight affected birds (seven ring necked parrots [5 males and 2 females], and one male African grey parrot) were submitted by the owner to the Pathology Laboratory, School of Veterinary Medicine, St. George's University for necropsy examination. Routine histopathology was performed on liver, lung, spleen, kidney, heart, brain, and intestines. Formalin-fixed liver was postfixed in $2.5 \%$ glutaraldehyde in $0.1 \mathrm{~m}$ cacodylate buffer and processed for transmission electron microscopy (TEM). Liver, lung, and spleen samples were subjected to culture on sheep blood agar and MacConkey media (Remel, Lennexa, KS, USA). The inoculated plates were incubated at $37^{\circ} \mathrm{C}$ for up to 72 hours, and checked for growth daily. Growth was recorded as pure (single colony type, confirmed with Gram-staining), or mixed (multiple colony types), and heavy (growth in all streak lines), light (growth only in primary streak inoculation area), or moderate (between light and heavy). Bacteria were identified to genus level using standard methods outlined by Quinn et al., (1994), and identified to species level using API bacterial identification strips (BioMerieux Inc., Durham, NC, USA). Antibiotic susceptibility tests were done using the standard disk diffusion method on Mueller-Hinton agar, and the inhibition zone sizes were interpreted as recommended by the Clinical and Laboratory Standards Institute (2008). The antibiotic discs used were ampicillin, $10 \mu \mathrm{g}$; amoxicillin-clavulanic acid, 30 $\mu \mathrm{g}$; cefoxitin, $30 \mu \mathrm{g}$; cephalothin, $30 \mu \mathrm{g}$; chloramphenicol, $30 \mu \mathrm{g}$; enrofloxacin, $5 \mu \mathrm{g}$; erythromycin, 15 $\mu \mathrm{g}$; gentamicin, $10 \mu \mathrm{g}$; penicillin, 10 units;

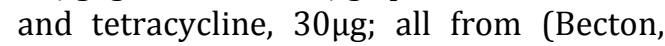
Dickinson and Co., Franklin Lakes, NJ, USA).

\section{Results}

Grossly, the liver and spleen were congested and enlarged. Diffuse red to tan mottling was present in the liver (Figures 1 and 2). The lungs were congested and heavy, and there were multifocal petechiae on the epicardial surface of the heart.

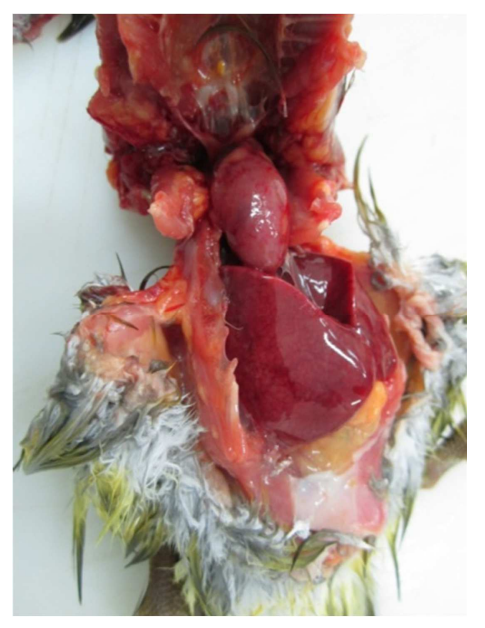

Figure 1. Ringneck Parrot in Dorsal Recumbency after Skinning; Moderate Hepatomegaly; Note the Rounded Margins and Red to Tan Mottling of the Liver 


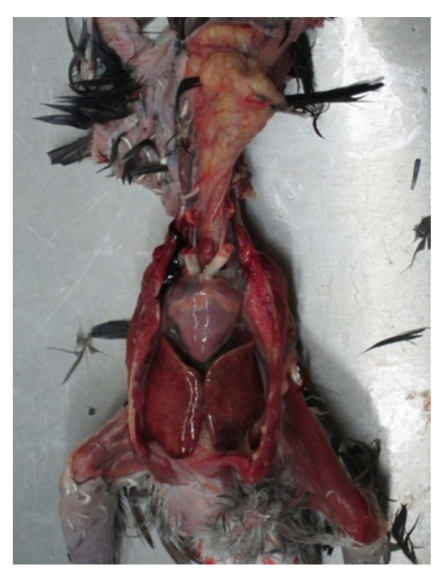

Figure 2. African Gray Parrot in Dorsal Recumbency after Skinning; Moderate Hepatomegaly; Note the Rounded Margins and Red to Tan Mottling of the Liver

Significant histopathologic findings included moderate to severe multifocal necrotizing hepatitis (Figure 3), mild to moderate interstitial pneumonia (Figure 4), mild lymphocytic nephritis, and mild lymphocytic myocarditis. There was nonsuppurative meningoencephalitis in one male ringneck parrot.

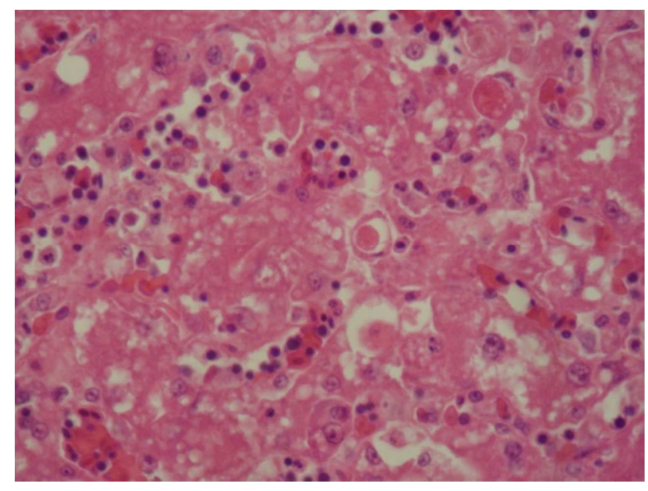

Figure 3. Ringneck Parrot; Liver; Marked Hepatocellular Degeneration and Necrosis with Mononuclear Inflammatory Cell Infiltration in the Hepatic Sinusoids, Necrotizing Hepatitis

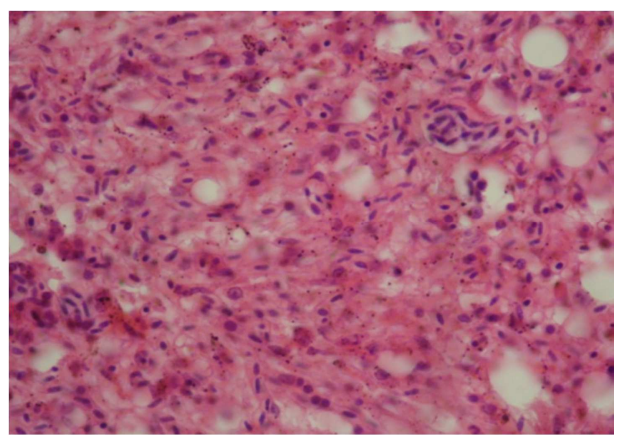

Figure 4. Ringneck Parrot; Lung; Marked Diffuse Congestion with Extensive Damage to the Air Capillaries and Inflammatory Cell Infiltration; Acute Interstitial Pneumonia 
Growth of bacterial colonies occurred only on blood agar, and none on MacConkey agar. Moderate to heavy growth of Staphylococcus spp. in pure culture occurred from $8 / 8$ liver samples, $7 / 8$ lung samples, and 5/8 spleen samples. Two samples of intestines were also positive for staphylococcus spp. All staphylococci were coagulase negative, and 2 isolates were identified as Staphylococcus lentus (98.6\% probability) using API Staph strips. One spleen sample yielded heavy, pure growth of a coagulase-negative Gram-positive coccus, which was later identified as Kocuria varians (98.7\% probability) using API staph strips. Isolates of staphylococci were susceptible to all of the 10 antimicrobial drugs tested against. Kocuria varians was resistant to tetracycline, and susceptible to the remaining 9 drugs.

PCR was negative for Pacheco's disease and transmission electron microscopy did not reveal intranuclear herpesvirus particles.

\section{Discussion}

Staphylococcus sciuri subsp. lentus gained species status as S. lentus in 1983 (Schleifer et al., 1983). Staphylococcus lentus is the predominant staphylococcal species associated with wild turkey feces. It has been shown as a potential causative agent of inflammatory reactions in the human respiratory tract (DeBoer et al., 2001). The role of this bacterium in psittacine bird is not known, and therefore worth further studies. Identification of Staphylococcus lentus using API Staph identification system has been reported to be $100 \%$ accurate (Stepanovic et al., 2005). Coagulasenegative Staphylococcus ssp. and Micrococcus spp. are commonly found in the feces of psittacine birds (Bangert et al., 1988). However, coagulase-negative species in lesions should not necessarily be interpreted as normal flora and ignored. Staphylococcal species can serve the dual role of commensals and opportunistic pathogens (Briscoe et al., 2009). Isolation in pure culture in high numbers from body sites which are not commonly colonized by these bacteria strongly indicates an association with disease. If bacteria have invaded tissues after death or if specimens have been improperly collected, a considerable variety of bacteria will be recovered unless selective media are used (Carter and Cole, 1990).

Kocuria spp. was previously classified as Micrococcus spp., and these colonize sites such as mucosa, oropharynx and skin of mammals. These opportunistic organisms have been isolated from cases of meningitis, sepsis, and pneumonia in humans (Tsai et al., 2010). The role of these bacteria in diseases of birds is not understood, although pure, heavy growth indicates a role. Necrotizing hepatitis occurs with most bacterial septicemias in birds and will usually be accompanied by lesions in the spleen, kidney, and lung. In birds with acute septicemias, the hepatic sinusoids become widened due to congestion, leukostasis, and swelling of Kupffer cells. Foci of necrotic hepatocytes may develop accompanied by an infiltration of heterophils in and around the necrotic area. The multifocal hepatic lesions observed in these birds are probably due to hematogenous infection of the liver by the staphylococci which were isolated from the liver of all the eight cases that were submitted for necropsy.

Table 1. Bacteria Isolated from Tissues of 8 Parrots

\begin{tabular}{|l|l|l|l|}
\hline Date & No. of birds & Isolate (No.) and positive samples & \% ID* \\
\hline $\begin{array}{l}23-2- \\
2012\end{array}$ & 1 & $\begin{array}{l}\text { Kocuria varians (1) (spleen) } \\
\text { Staphylococcus spp. (1) } \\
\text { (coagulase-negative) (liver) }\end{array}$ & 98.7 \\
\hline $3-3-2012$ & 5 & $\begin{array}{l}\text { Staphylococcus spp. (5) (coagulase- } \\
\text { negative) (Liver, lung, spleen) }\end{array}$ & Not identifiable to species level** \\
\hline $\begin{array}{l}29-3- \\
2012\end{array}$ & 2 & $\begin{array}{l}\text { Staphylococcus lentus (2) } \\
\text { (liver, lung, intestine) }\end{array}$ & 98.6 \\
\hline
\end{tabular}

*Probability according to API database

**Not identifiable to species level using API identification system

Muhammad I. Bhaiyat, Harry Hariharan, Alfred Chikweto, Keshaw Tiwari, Ravindra N. Sharma and Yoshiyasu Kobayashi (2013), International Journal of Veterinary Medicine: Research \& Reports, DOI: 10.5171/2013.149634 


\section{Acknowledgements}

The authors gratefully acknowledge the technical assistance of Erica BrathwaiteSylvester.

\section{References}

Bangert, R. L., Cho, B. R., Widders, P. R., Stauber, E. H. \& Ward, A. C. (1988). "A Survey of Aerobic Bacteria and Fungi in the Feces of Healthy Psittacine Birds," Avian Diseases 32 (1) 46-52.

Barao Da Cunha, M., Correia, J. J., Fagulha, T., Fevereiro, M., Plleteiro, M. C., Vollrath, G. \& Kaleta, E. F. (2007). "Pacheco's Parrot Disease in Macaws of the Lisbon's Zoological Garden. Description of an Outbreak, Diagnosis and Management, Including Vaccination," Deutsche Tierarztliche Wochenschrift. 114 (11) 423428.

Bistyak, A., Kecskemeti, S., Glavits, R., Tischler, I., Nagy, S. T., Kardos, G. \& Kiss, I. (2007). "Pacheco's Disease in a Hungarian Zoo Bird Population: A Case Report," Acta Veterinaria Hungarica 55 (2) 213-218.

Bounous, D. I., Schaeffer, D. O. \& Roy, A. (1989). "Coagulase-Negative Staphylococcus Sp Septicemia in a Lovebird," Journal of the American Veterinary Medical Association 195 (8) 1120-1122.

Briscoe, J. A., Morris, D. O., Rosenthal, K. L., Shofe, F. S. \& Rankin, S. C. (2009). "Evaluation of Mucosal and Seborrheic Sites for Staphylococci in Two Populations of Captive Psittacines," Journal of the American Veterinary Medical Association. 234(7) 901-905.

Carter, G. R. \& Cole, Jr. J. R. (1990). Diagnostic Procedures in Veterinary Bacteriology and Mycology, Academic Press, Inc., San Diego, California. Pp 19-39.

Clinical and Laboratory Standards Institute. (2008). 'Performance Standards for Antimicrobial Disk and Dilution Susceptibility Tests for Bacteria Isolated from Animals,' Approved Standard - Third Edition CLSI Document M31-A3, Wayne.

de Freitas Raso, T., Godoy, S. N., Milanelo, L., De Souza, C. A., Matuschima, E. R., Araujo Jonior, J. P. \& Pinto, A. A. (2004). "An Outbreak of Chlamydiosis in Captive BlueFronted Amazon Parrots (Amazona Aestiva) in Brazil," Journal of Zoo and Wildlife Medicine. 35 (1) 94-96.

Deboer, L. R., Slaughter, D. M., Applegate, R. D., Sobieski, R. J. \& Crupper, S. S. (2001). "Antimicrobial Susceptibility of Staphylococci Isolated from the Faeces of Wild Turkeys (Meleagris Gallopavo)," Letters in Applied Microbiology. 33(5) 382386.

Foldenauer, U., Rusch, M., Simova-Curd, S., Nitzl, D., Hoop, R. K. \& Hatt, J. M. (2009). "Endocarditis due to Lactobacillus Jensenii in a Salvin's Amazon Parrot (Amazona Autumnalis Salvini)," Avian Pathology. 38 (1) $55-58$.

Galindo-Cardiel, I., Opriessnig, T., Molina, L. \& Juan-Salles, C. (2012). "Outbreak of Mortality in Psittacine Birds in a Mixed Species Aviary Associated with Erysipelothrix Rhusiopathiae Infection," Veterinary Pathology. 49 (3) 498-502.

Hermans, K., Devriese, L. A., De Herdt, P., Godard, C. \& Haesebrouck, F. (2000). "Staphylococcus Aureus Infections in Psittacine Birds," Avian Pathology. 29 (5) 411-415.

Quinn, P. J., Carter, M. E., Markey, B. \& Carter, G. R. (1994). "Clinical Veterinary Microbiology," Wolfe Publishing, Mosby Year Book Europe Limited, London. Pp 118126.

Schleifer, K. H., Geyer, U., Kilpper-Balz, R. \& Devriese L. A. (1983). "Elevation of Staphylococcus Sciuri Subsp," Lentus (Kloos Et Al) To Species Status: Staphylococcus Lentus (Kloos Et Al) Comb. Nov," Systematic and Applied Microbiology. 4 (3) 382-387. 
Stepanovic, S., Dakic, I., Morrison, D., Hauschild, T., Jezek, P., Petras, P., Martel, A., Vukovic, D., Shittu, A. \& Devriese, L. A. (2005). "Identification and Characterization of Clinical Isolates of Members of the Staphylococcus Sciuri Group," Journal of Clinical Microbiology. 43 (2) 956-958.

Tarello, W. (2001). "Chronic Fatigue and Immune Dysfunctions Syndrome Associated with Staphylococcus Spp. Bacteremia Responsive to Thiacetarsamide Sodium in Eight Birds of Prey," Journal of Veterinary Medicine Series B - Infectious Diseases and Veterinary Public Health. 48 (4) 267-281.

Tsai, C.- Y., Su, S., Cheng, Y.- H., Chou, Y., Tsai, T.- H. \& Lieu, A.- S. (2010). "Kocuria Varians Infection Associated with Brain Abscess: A Case Report," Bio Med Central Infectious Diseases 10: 102.

Ward, M. P., Ramer, J. C., Proudfoot, J., Garner, M. M., Juan-Salles, C. \& Wu, C. C. (2003). "Outbreak of Salmonellosis in a Zoologic Collection of Lorikeets and Lories (Trichoglossus, Lorius, and EosSpp.)," Avian Diseases. 47 (2) 493-498. 